\title{
Spiking variability: Theory, measures and implementation in Matlab
}

\author{
Eric S. Kuebler ${ }^{\mathrm{E}}$, a, Jean-Philippe Thivierge a \\ a School of Psychology, University of Ottawa
}

\begin{abstract}
The quantification of spiking variability is prevalent to many questions in neuroscience. In this review, several measures of variability are presented, as well as algorithms for implementing analyses including: spike rates and Fano factor, inter-spike intervals, coefficient of variation and local variation, autocorrelation, period histograms, a synchrony index (vector strength), and finally post-stimulus time histograms. Some of the techniques show significant overlap; however, each measure is qualitatively unique and can be tuned to the researchers needs.
\end{abstract}

Keywords " Spiking variability, time-series data, neuronal networks, Matlab

Đeric.s.kuebler@gmail.com

\section{Introduction}

Neurons emit action potentials or 'spikes' which are said to carry information to downstream neurons and throughout the brain (Reike, Warland, van Steveninck, \& Bialek, 1999). From the perspective of a single neuron embedded in a network, spike timing can be a function of intrinsic (i.e., ionic currents) and extrinsic factors (i.e., a function of the integration performed by a neuron receiving excitatory pre-synaptic inputs); however, most of the variation is a product of synaptic inputs (Mainen \& Sejnowski, 1995). Thus the signal emitted by a neuron has the potential to be unreliable from a computational perspective this presents an issue (Pouget, Dayan, \& Zemel, 2000). Spike trains are highly variable (i.e., van Steveninck, Lewen, Strong, Koberle, \& Bialek, 1997) and numerical simulations have shown that the signal emitted by spiking neurons is chaotic in the sense that it is non-repetitive and sensitive to small perturbations (Kuebler, Bonnema, McCorriston, \& Thivierge, 2013; Thivierge, 2008; Thivierge \& Cisek, 2008). Neurons, however, and the networks they are embedded in, have the daunting task of deciphering neuronal codes and processing this rich and diverse flow of information.

The quantification of spiking variability is an important goal in neuroscience: researchers have used measures of variability to broaden our understanding of brain function. For instance, during the development of neuronal circuits, significant importance has been attributed to a neuron's intrinsic variability - which gives it the ability to generate highly variable spike patterns (Gabbiani \& Cox, 2010). Consistent with this idea, dentate gyrus adult newborn cells (i.e., progenitor cells that reach maturation during an individual's adulthood) spike more often and spontaneously than those neurons already integrated into the network (Laplagne, Zhao, Lombardi, Ribak, et al., 2008). These two studies characterize examples where the numerical assessment of spiking variability has enlightened our understanding of brain function and both demonstrate a fundamental principle in neuroscience, namely: those that fire together wire together (Hebb, 1949).

Statistics representing spiking variability can be used to derive the unique characteristics of neurons in the brain (Gabbiani \& Cox, 2010). One famous example comes from recording the visual cortex of felines. Hubel and Wiesel (1959) showed that the spike rate of a single neuron increased when presented with stimulus of a preferred orientation angle (i.e., black bar on a white background). The study by Hubel and Wiesel (1959) is a hallmark in neuroscience literature and was largely based on the analysis of spike rates. More recently, inter-spike interval (ISI) histograms were used to quantify the differences in bursting behaviour between projection and local interneurons, researchers then went on to explore the functional implications of these findings (Lei, Reisenman, Wilson, Gabbur, \& Hildebrand, 2011). These examples characterize two of many instances (i.e., Bosman, Schoffenlen, Brunet, Oostenveld, Bastos, et al., 2013; Dupret, O'Neill, Csicsvari, 2013) where simple analyses of spiking variability served as a foundation from which to explore and advance our understanding of the 
characteristic differences between neurons.

In addition to single neuron analyses, networks of spiking neurons can be characterized using spiking variability. Simple analyses, such as spike rates, Fano factors (a measure of variability in spike rate distributions), and correlations, have been used to show that clustered neuronal networks behave chaotically in two ways, namely: variations in spike rate and timing (Churchland \& Abbott, 2012; Litwin-Kumar \& Doiron, 2012). In addition, using the Fano factor, investigators showed that the presentation of stimuli reduced variability (Churchland, Yu, Cunningham, Sugrue, Cohen, et al. 2010) and in numerical simulations, Litwin-Kumar and Doiron (2012) show that the reduction in variability only occurs when networks are clustered (i.e., excitatory cells are clustered together). Both studies use the spike rate and Fano factor to analyze spiking activity, further they show that it is possible to derive the characteristics of neural networks using simple measures.

Finally, variability measures can be used to examine the possibility of candidate neural coding. Two codes have attracted the attention of researchers: (1) rate or independent coding, and; (2) temporal or correlated coding (Reike et al., 1999; Dayan \& Abbott, 2001). Researchers have shown that both rate (i.e., London, Roth, Beeren, Hausser, \& Latham, 2010) and temporal coding (i.e., Adler, Finkes, Katabi, Prut, \& Bergman, 2013) are relevant to information processing (see Ainsworth, Lee, Cunningham, Traub, Kopell et al., 2012). It is possible that some areas of the brain encode information relative to the rate of spiking activity; while others encode information based on correlated inputs and therefore rely on temporal coding. In addition to rate and temporal coding, researchers have suggested these codes can be coupled with a phase code - where information is added to the candidate code when considered relative to the phase of ongoing oscillations (Kayser, Montemurro, Logothetis, \& Panzeri, 2009; Turesson, Logothetis, \& Hoffman, 2012).

The previous paragraphs have shown that numerous questions related to brain function can and have been answered or at least approached critically using simple analyses of variability in spiking activity. Spiking variability can be used to derive the characteristics of neurons, neural networks, and the neural codes. Many of the measures presented below can be used with stimuli. This possibility, however, should be evaluated with regard to your specific hypotheses.
Since a number of measures of spiking variability exist, many non-linear measures (i.e., information theory and decoding algorithms) are not discussed with the exception of local variation. Action potential events can be non-linear events - thus some of the measures may present a slight underestimation of actual variability. Many of the measures presented can be applied to a wide range of multi-disciplinary questions. For example, the coefficient of variation $\left(C_{V}\right)$ is commonly used in probability fields, and the Fano factor is used in physics for particle detection (Pennini \& Plastino, 2010). Here, we present the quantification of variability by broad stroke measures (i.e., spike rate) such as spike rates and Fano factors, as well as more precise measures (i.e., spike time) such as inter-spike intervals (ISIs), $C_{V}$, local variation ( $\left.L v\right)$, autocorrelation, period histograms, a synchrony index (vector strength - VS), and post-stimulus time histograms (PSTH).

\section{Measures of Spiking Variability}

\section{Spike rate and Fano factor}

Spike rates are a broad stroke measure of a neurons activity over a duration of time measured in Hz. Spike rates do not measure variability, they are, however, commonly used to quantify a neural response over a given duration of time $T$. When a neuron's spiking activity is represented as a vector of spike times $t_{1}, t_{2}$, $t_{3} \ldots t_{N}$, the rate is defined by

$$
R=\frac{N}{T}
$$

where $N$ is the total number of spikes over a given duration $T$ (some unit of time). $T$ can be 'binned,' where, for instance, $100 \mathrm{~ms}$ of spiking activity is reduced to a 50 bit vector by using $2 \mathrm{~ms}$ bins and having '1's denote spikes, '0's no spikes (i.e., Kayser et al., 2009). Binning, or down sampling, is not the subject of this tutorial but can be useful (full explanation and implementation schemes are described in: Reike et al. 1999; Dayan \& Abbott, 2001). Spike rates can be computed as a function of single neurons across trials, or over a network, thus generating a distribution of rates with variability (i.e., $R_{1}, R_{2}, R_{3}, \ldots R_{f}$, where $J$ is the total number of neurons or trials). Fano factors $F$ are a measure of the dispersion in a distribution. Thus Fano factors $F$ are commonly used to measure the dispersion in the spike rate distribution, this makes $F$ a broad stroke measure of variability in the signal produced by 
Figure 1 Measures of spiking variability. A. Cartoon schematic of a spike train. Black vertical lines are spikes across time. Red arrow heads denote the duration of time $\Delta t$ between two spikes - termed an inter-spike interval (ISI). B. Cartoon schematic of an autocorrelation generated from the spike trains of a neuron A. Black horizontal lines are spikes across time. Red box shows spikes aligned at $\Delta t=0$, these spikes are used in generating the autocorrelation function. C. A spike raster depicted as binned activity. Red boxes are single bins (i.e., matrices) of spiking activity that are summed across neurons generating a network response. Notice that the time bins are the same duration as the period of the frequency of interest (i.e., $100 \mathrm{~ms}$ ). Grey box on wave below captures the full cycle of the oscillation. D. Spike raster depicting the response of a network of neurons to a stimulus pattern. For this example the spikes were produced by a network where oscillations were injected $(10 \mathrm{~Hz}-5 \mathrm{~mA})$. Red circles denote a random depolarizing event delivered to a subset of cells - the stimulus pattern. Gray box is the post-stimulation response window $(100 \mathrm{~ms})$ stored for offline analysis of post-stimulus histogram.
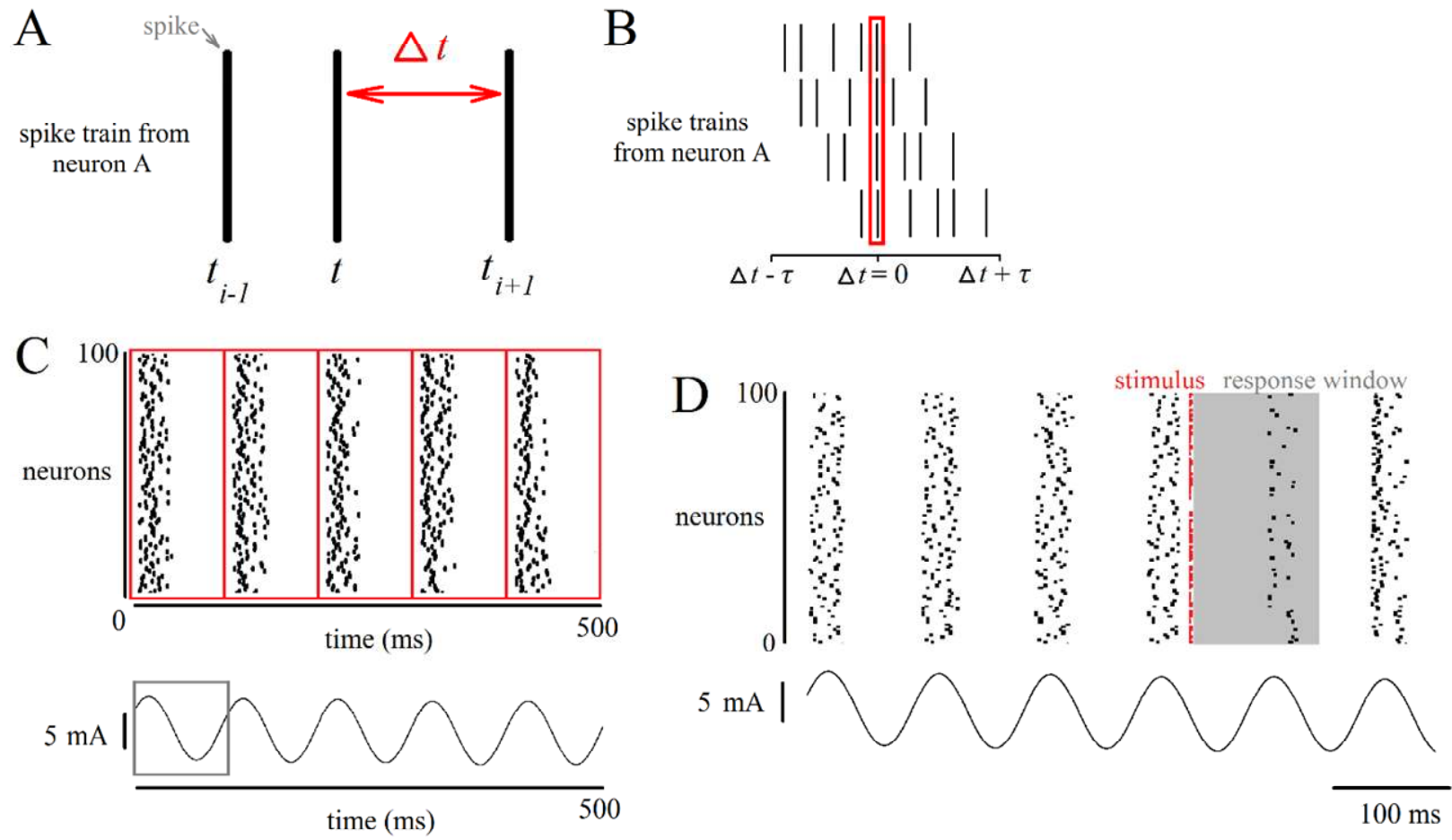

a neuron or neuronal network. For random spike rates $R_{J}, F$ is equal to 1 ; by comparison, for constant spike rates $R, F$ is equal to zero (Dayan \& Abbott, 2001). $F$ quantifies the amount of variability in the spiking activity relative to the mean spike rate, and is defined by

$$
F=\frac{1}{\bar{R}} \sqrt{\frac{1}{J-1} \sum_{j=1}^{J}\left(R_{j}-\bar{R}\right)^{2}}
$$

where $R_{j}$ is a given spike rate, $J$ is the total number of spike rates, and $\bar{R}$ is defined by

$$
\bar{R}=\frac{1}{J} \sum_{j=1}^{J} R_{j}
$$

Since $R$ can be computed for several trials or neurons in a network, a single value $F$ can describe variability for a single neuron or neuronal network thus making it flexible to the researcher's interests. Since $R$ is a summation of $N$ spikes over time $T, R$ is a macro scale measure of spiking activity and $F$ is a macro scale measure of variability, next we examine measures of variability in terms of precise spike timing.

\section{Inter Spike Intervals (ISIs)}

An ISI measures the latency $\Delta t$ between precise spike times (Figure 1A - Dayan \& Abbott, 2001). ISIs can be computed for every single spike (minus one) or a percentage of spikes. When a neuron's spiking activity is arranged in a vector of spike times $t_{1}, t_{2}, t_{3} \ldots t_{N}$, the $t$ th ISI is defined by

$$
\Delta t_{i}=t_{i+1}-t_{i}
$$


where $t$ represents a given spike time. Intervals are then counted, generating a distribution of ISIs for single neurons; or by comparison, a network of neurons. Differences in the temporal spike patterns emitted by single neurons (i.e., Lei et al. 2011) or networks of neurons (i.e., Wallace, Benayoun, van Drongelen, \& Cowan, 2011) can become notably visible through ISI distributions. The characteristics of the ISI distribution can be established by examining the $C_{V}$ and $L v$. For a more detailed treatment of the relation between rates and intervals see Lemon (2014).

\section{Coefficient of Variation (Cv)}

The $C_{V}$ is a measure of the dispersion in the ISI distribution (Dayan \& Abbott, 2001). When we calculated $F$ we use variance, by comparison, the $C_{V}$ is computed using the standard deviation. For sufficiently long Poisson processes (where neurons spike at a fixed rate and spikes times are random) the $C_{V}$ is 1 ; by comparison, for a constant $\Delta t$ the $C_{V}$ is zero (Dayan \& Abbott, 2001). Using the vector of spike times $t_{1}, t_{2}$ $t_{3} \ldots t_{N}$, the $C_{V}$ is defined by

$$
C v=\sqrt{\frac{1}{N-2} \sum_{i=1}^{N-1}\left(\Delta t_{i}-\overline{\Delta t}\right)^{2}} / \overline{\Delta t}
$$

where $N$ is the total number of spikes (minus 1 in the equation because intervals are a measure between two points in time, thus there are $N-1$ intervals), $\Delta t$ is a given ISI defined by equation (4), and $\overline{\Delta t}$ is defined by

$$
\overline{\Delta t}=\frac{1}{N-1} \sum_{i=1}^{N-1} \Delta t
$$

Since ISIs can be computed for a single neuron, or an entire network, the $C_{V}$ can be computed for the entire network generating a scalar value, or computed for each neuron's ISI distribution, generating a distribution of $C_{V}$ for the entire network.

\section{Local Variation (Lv)}

Closely related to the $C_{V}$ is the measure of local variation $(L V)$. The measurement of $L V$ is a relatively new method of examining the ISI distribution (Shinomoto \& Shima, 2003) and has been met with some criticism (i.e., Ikeda, 2005; Ponce-Alvarez, Kilavik, \& Riechle, 2010). The $L V$ is said to be sensitive to the intrinsic characteristics of neurons since it measures the step-wise variability of ISIs (Shinomoto \& Shima, 2003). $L_{V}$ is a non-linear measure, and like the $C_{V}$, is based on ISIs, sufficiently long Poisson processes have a $L v$ equal to 1 , and for constant $\Delta t L v$ equals zero. When a neurons spike train is defined by a vector $t_{1}, t_{2}, t_{3} \ldots t_{N}$ the $L v$ is defined by

$$
L v=\frac{3}{N-2} \sum_{i=1}^{N-1} \frac{\left(\Delta t_{i}-\Delta t_{i+1}\right)^{2}}{\left(\Delta t_{i}+\Delta t_{i+1}\right)^{2}}
$$

where $N$ is the total number of spikes, and $\Delta t$ is a given ISI. The quadratic difference between ISIs (i.e., the second term in the numerator) is multiplied by 3 so that Poisson processes equal to $\sim 1$ (Shinomoto \& Shima, 2003).

\section{Autocorrelation}

Autocorrelation is one way to measure how well a signal is correlated with itself (Reike et al., 1999). Using an autocorrelation, we quantify the self-similarity of a neurons signal as a function of the time lag $\tau$. In the context of this discussion, we use correlations to examine the periodicity in a single neuron's spiking behaviour. To do this, we take a given spike train, randomly pick a spike, then align spikes such that for each subsequent row, neuron A's spike train is shifted to the left and the succeeding spikes are aligned at $\Delta t=$ 0 (Figure 1B - Reike et al., 1999). The proportion of all aligned spike trains is the autocorrelation function, these spikes should be aligned at $\tau=0$ (measured in $\mathrm{ms}$ ). Thus turning the proportion into a probability creates a peak of $p=1$ in the autocorrelation at $\tau=0$. The duration of recording included in the autocorrelation is left to the researcher. One way of determining the size of the window $\tau$ (or duration of time) is to think of what frequencies you expect to see in the data. In the example provided below, $\tau$ is equal to $400 \mathrm{~ms}$, so that a periodic signal as a slow as $0.4 \mathrm{~Hz}$ could be detected. There is the added option of normalizing the autocorrelation function to the spike rate which produces a conditional rate (see Reike et al. 1999). Here variability is measured across time, you would look at some measure of error (i.e., SEM is plotted in the upcoming examples) around the mean at a given time-step $\tau$.

\section{Period Histogram}

Periodic inputs, injected into single neurons, have been shown to generate periodic outputs (i.e., Mainen \& Sejnowski, 1995), and period histograms can be used to show the periodicity in a signal elicited by an input. Period histograms visually depict the precise timing of 
spikes relative to the phase of an oscillating current. To generate a period histogram, spiking activity is binned where bin width is set to the duration of the period of the oscillating current (i.e., red boxes - Figure 1C). In this scenario, we define a periodic input using a sine wave current that reaches peaks between minimum and maximum limits when amplitude is added (i.e., 5 $\mathrm{mA})$. Spiking activity within each bin is counted, and the counts are averaged across a given number of bins (i.e., in the example five cycles are binned, and averaging across the bins would create the period histogram). Variability is measured in the same fashion as autocorrelation, that is to look at variability across time, you would look at some measure of error (i.e., SEM is plotted in the upcoming examples) around the mean at a given time step.

\section{Synchronization Index (Vector Strength)}

Period histograms visually depict periodicity; by comparison, synchronization indices such as vector strength $V S$ are useful in numerically depicting the degree of synchrony between two signals (Goldberg \& Brown, 1969; Chang, Bao, Imaizumi, Schreiner, \& Merzenich, 2005). VS quantifies synchrony by taking precise spike times and determining their phase relation to a sine wave. There are many useful ways to define and compute synchrony (i.e., across neurons, across networks, across conditions, etc.); here, we compute the degree of synchrony between an input (i.e., oscillating current with a period $c$ ) and the signal emitted by a neuron in the form of spike times $t_{1}, t_{2}$, $t_{3} \ldots t_{N}$. For completely Poisson processes $V S$ is zero; if the signals are synchronized, however, $V S$ is one. The Rayleigh statistic $(2 M V S])$ can be used to assess the significance of $V S$, more specifically critical values of 5.991 for $\alpha=0.05$, and 13.816 for $\alpha=0.001$ can be compared to establish significance (Chang et al. 2005). When spike times are arranged in a vector $t_{1}, t_{2}, t_{3} \ldots t_{N}$ $V S$ is defined by

$$
V S=\frac{1}{N} \sqrt{x^{2}+y^{2}}
$$

where $N$ is the total number of spikes, $x$ is defined by

$$
x=\sum_{i=1}^{N} \cos \theta_{i}
$$

$y$ is defined by

$$
y=\sum_{i=1}^{N} \sin \theta_{i}
$$

and $\theta_{i}$ is defined by

$$
\theta_{i}=2 \pi \frac{\bmod \left(t_{i} / c\right)}{c}
$$

where $t$ is the spike time for $i^{t h}$ neuron/channel, and $c$ is the period of the input wave. Here the spike time $t_{i}$ modulo the oscillations period $c$ is the moment within the period (between 0 and $2 \pi$ ) when the neuron spikes and $\theta_{i}$ is the phase. Thus, this measure is an indication of the strength of the coherence between the injected signal and one emitted by a neuron in response. Variability is measured in the same way as autocorrelation and period histograms, that is to look at variability across time, you would look at some measure of error (i.e., SEM is plotted in the upcoming examples) around the mean at a given frequency.

\section{Post-Stimulus Time Histograms (PSTH)}

PSTHs measure the timing of spikes relative to a stimulus presentation (Reike, et al., 1999; Dayan \& Abbott, 2001). Post-stimulus (i.e., red spikes denote stimulus - Figure 1D) responses are captured (gray box - Figure 1D) and counted so that researchers can visually characterize the neural response. We now have the added option of turning the counts into proportions or probabilities by dividing the value of each data point by the sum of all data points; in the forthcoming example this was not done because there were only ten stimulus presentations. It is recommended that stimuli be repeatedly presented such that a probability distribution can be generated because spike times are quite variable across repetitions of the same stimulus (Mainen \& Sejnowski, 1995). Variability is measured in the same fashion as autocorrelation, VS, and period histograms, that is to look at variability across time, you would look at some measure of error (i.e., SEM is plotted in the upcoming examples) around the mean at a given time step.

\section{Examples of Spiking Variation}

We have generated data to provide some visual examples that will enhance the end user's understanding of the measures - see Matlab Functions and Example Data below. To generate this data we used 
Figure 2 - Examples of spiking activity. A. Cartoon schematic of an oscillation with an amplitude of zero. The black curve indicates what amplitude would be injected at that time step. B. Cartoon schematic of an oscillation with a frequency of $10 \mathrm{~Hz}$ and an amplitude of $5 \mathrm{~mA}$. C. Spike raster depicting the response of a network of spiking neurons. For this example the spikes were produced by a network without an oscillation injected. D. Spike raster depicting the response of the network. For this example the spikes were produced by a network with an oscillation injected $(10 \mathrm{~Hz}$ - $5 \mathrm{~mA}$ ). E. Similar network to $\mathrm{C}$ responding to a stimulus pattern. Red circles denote a random depolarizing event delivered to a subset of cells - the stimulus pattern. F. Similar network to D responding to a stimulus pattern $(10 \mathrm{~Hz}$ $-5 \mathrm{~mA})$.
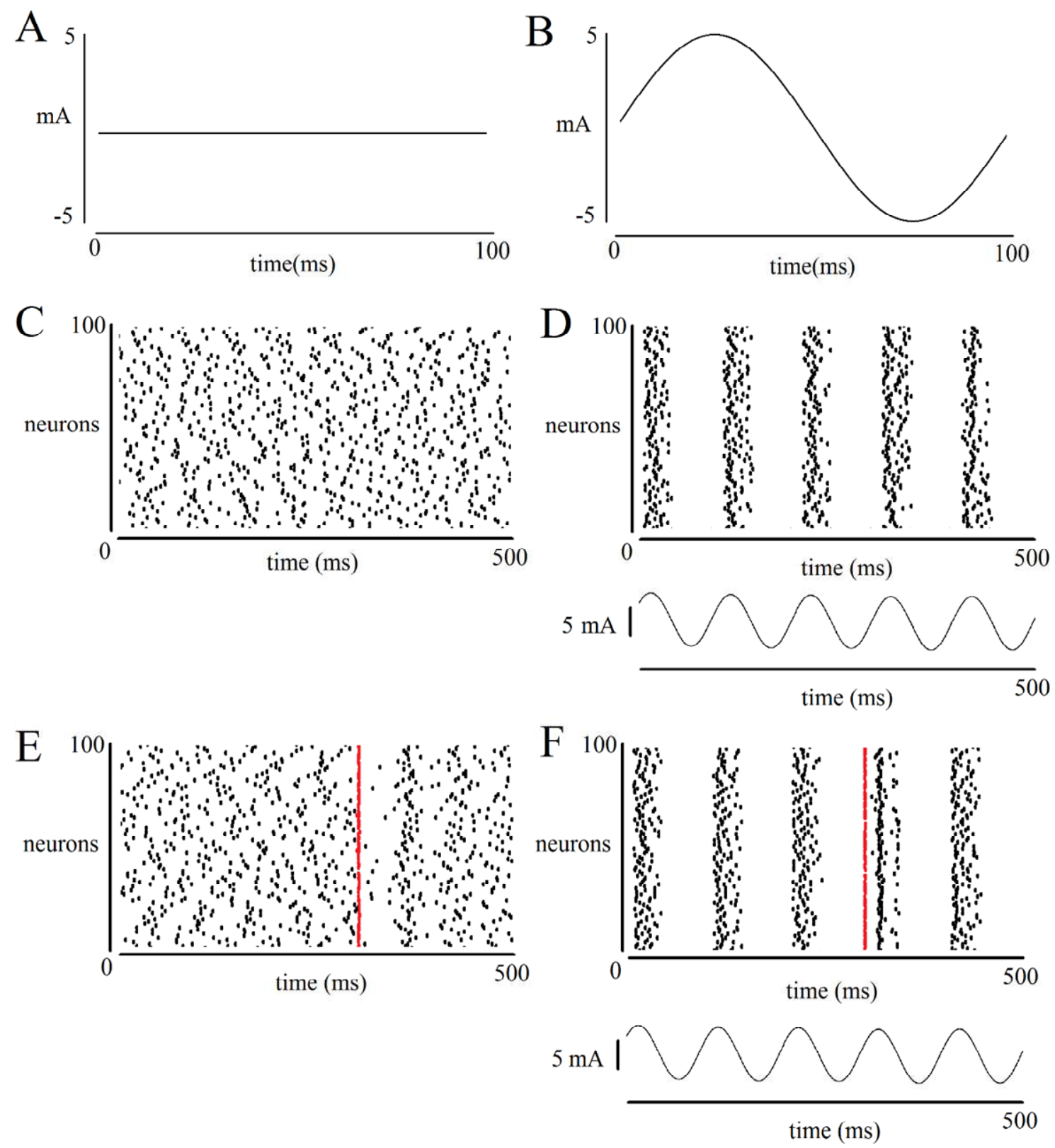

a computational model of interacting cells that emit action potentials (i.e., Gerstner \& Kistler, 2008; Kuebler, Bonnema, McCorriston, \& Thivierge, 2014). The spiking data is passed to each function in the form of a matrix $\mathbf{X}$ of any size where the rows represent neurons (i.e., $n_{1}$, $n_{2}, n_{3} \ldots n_{N}$ ) or trials (i.e., $k_{1}, k_{2}, k_{3} \ldots k_{N}$ ) and columns represent time (i.e., $t_{1}, t_{2}, t_{3} \ldots t_{N}$ ). Within $\mathbf{X}$, ' 1 's must denote spikes, and ' 0 's denote silence. Here we define 
Figure 3 - Examples of spiking variability. Left column: figures represents a neuronal network without oscillations. Right column: figures represents a neuronal network with oscillations ( $10 \mathrm{~Hz}-5 \mathrm{~mA})$. A1. \& A2. Spike rate distribution and Fano factor $(F)$. B1. \& B2. Inter-spike interval distribution and coefficient of variation $(C V)$. C1. \& C2. Local variation distribution. D1. \& D2. Autocorrelation function. Red shading represents standard error of measurement (SEM). E1. \& E2. Period histogram. Red shading represents standard error of measurement (SEM). F1. \& F2. Vector strength.
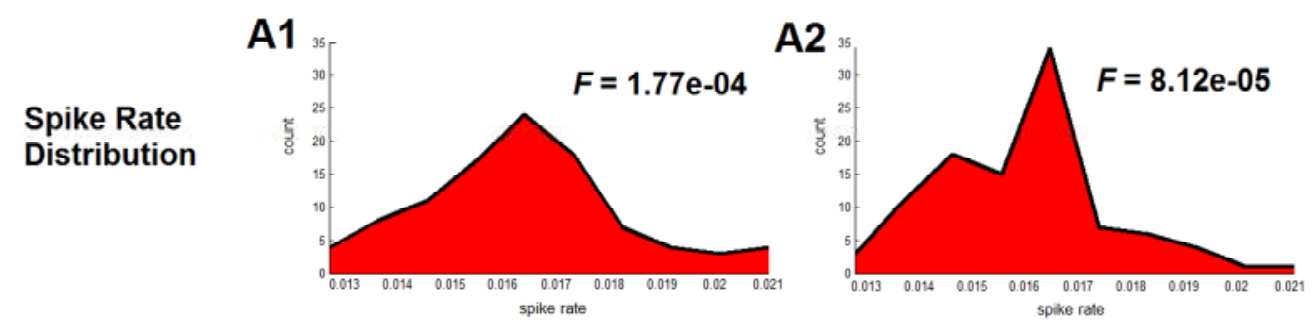

ISI

Distribution
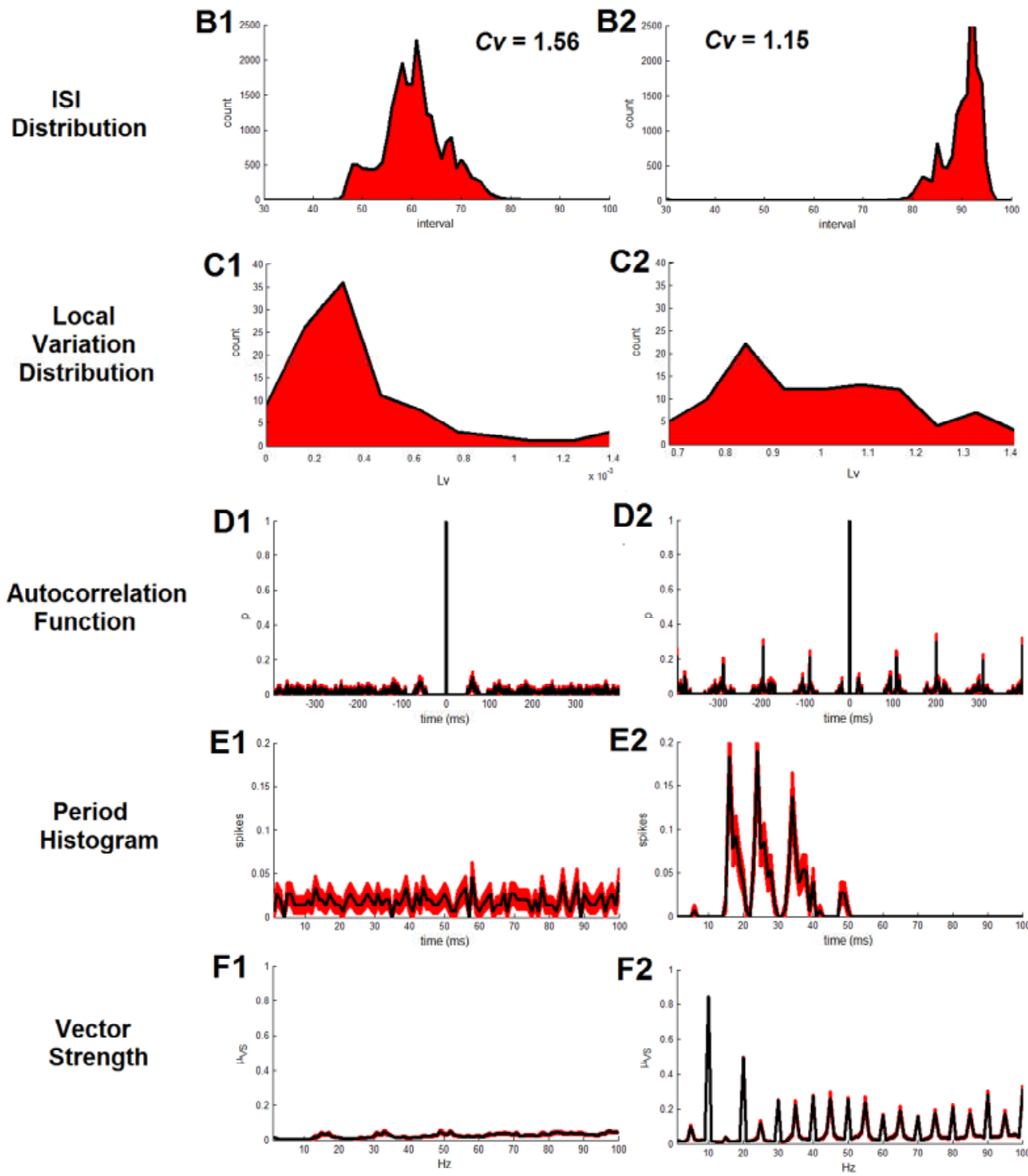

D2
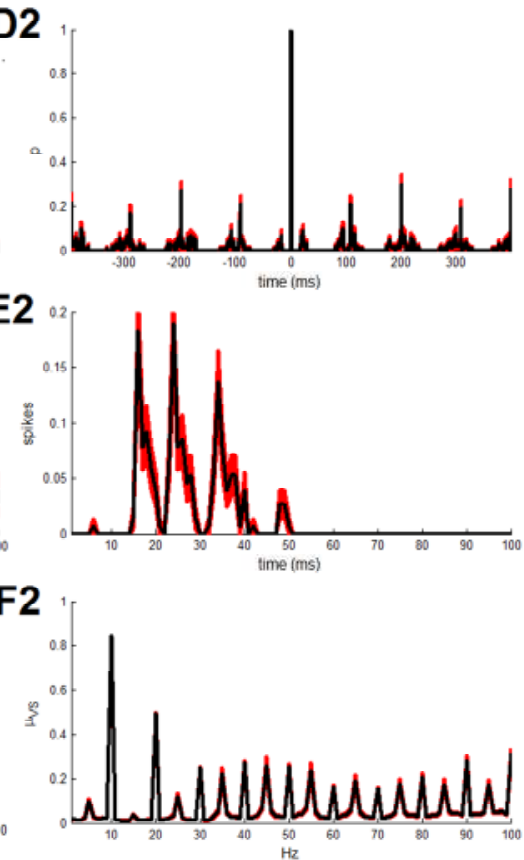

oscillations as a sine wave current that reaches peaks between minimum and maximum limits when Figure 2B). For clarity, we simulated spiking activity to capture four conditions, neuronal networks: [1] without oscillations or stimulus injected (i.e., Figure amplitude is added (i.e., compare Figure $2 \mathrm{~A}$ versus 
2C); [2] with oscillations and without stimuli (i.e., Figure 2D); [3] without oscillations and with stimuli (i.e., Figure 2E); as well as, [4] with oscillations and with stimuli (i.e., Figure $2 \mathrm{~F}$ ). We define stimuli as random depolarizing events delivered to a subset of cells (i.e., red circles in Figure 2E \& 2F). To enhance the readers understanding we present some examples for the following measures: spike rate, Fano factor, ISIs, $C_{V}$, $L V$, autocorrelations, period histograms, $V S$, and PSTHs.

To examine the spiking activity in terms of global measure (i.e., long durations of time) we look at spike rates and the Fano factor $(F)$. For this analysis we compare two conditions: [1] the network without oscillations (Figure 2A); and, [2] the network with oscillations (i.e., $10 \mathrm{~Hz}, 5 \mathrm{~mA}$ - Figure 2B). Results of this analysis show that networks without oscillations had a normal distribution of spike rates that was not highly variable, $F=1.77 \mathrm{e}-04$ (Figure 3A1). By comparison, networks with oscillations had a spike rate distribution that was normal; but less variable, $F=$ 8.12e-05 (Figure 3A2). This example shows how spike rates and $F$ can be used to quantify variability in spiking activity.

To investigate the temporal patterns in spiking we can examine latencies between spikes emitted by a neuronal network, for this we utilize the ISI distribution and $C_{V}$. Results of this analysis show that the network without oscillations had a normal distribution of intervals, and the $C_{V}=1.56$ indicates that the spikes were highly variable (black curve - Figure 3B1). By comparison, the network with oscillations $(10 \mathrm{~Hz}-5$ $\mathrm{mA}$ ) had a normal distribution that was shifted and skewed towards longer intervals and the $C_{V}=1.15$ indicates spike times were less variable (black curve Figure 3B2). This example depicts the utility of ISIs in showing that the temporal pattern within two signals is different.

Another way to examine variability in the ISI distribution is to look at the local variation $(L V)$ distribution. Results of this analysis show that networks without oscillations had a $L v$ distribution that was normal; yet positively skewed (Figure 3C1). By comparison, networks injected with oscillations had a distribution that was normal; but less heavily skewed in comparison to networks without oscillations (compare Figure 3C1 versus Figure 3C2). This example shows how variability, in the form of $L v$ can be used to characterize differences between neuronal networks.

To further examine the periodicity in the signal emitted by single neurons in the network we compare the autocorrelation function for the two conditions above. Results of this analysis show that the autocorrelation function for cells in a network without oscillations appeared stochastic - indicated by the curve appearing largely flat (Figure 3D1). By comparison, the autocorrelation function for cells in a network with oscillations injected showed greater periodicity - indicated by well-defined peaks and troughs (Figure 3D2). These results are consistent with the spike rasters presented in Figure 2C and D: [1] cells in the network without oscillations spiked randomly; [2] cells in the network with oscillations spiked with a periodic rhythm. We can examine the variability in the function of each network by looking at the shaded area (red - Figure 3D1 \& 3D2). Within these networks the variability is low, this is probably because there was low levels of heterogeneity between cells (i.e., reversal potential, capacitance, time constants, etc.). This example shows how autocorrelations can be useful in quantifying the periodicity and variability in the signal emitted by single cells.

To assess the periodicity in the signals emitted by a network of interacting cells we examine period histograms. Results show that the network without an oscillation injected did not respond with any periodicity (Figure 3E1) - this aligns well with previous results (i.e., flat autocorrelation function - Figure 3D1). By comparison, networks injected with oscillations were more periodic and most spiking occurred during the peak of the injected oscillation (Figure 3E2) - this is consistent with previous results (i.e., periodicity in the autocorrelation function - Figure 3D2). We can examine the variability by looking at the shaded red area, here we see that spiking activity was less variable when oscillations were injected (compare Figure 3E1 versus Figure 3E2). These examples depict how period histograms can be useful in showing the variation in network responses to periodic input.

Next we examine the $V S$ to quantify the level of synchronization to frequencies of an oscillation. Results of this analysis show that the network without oscillations was not synchronized with any frequencies - indicated by a flat curve (Figure 3F1). By comparison, the network with an oscillation injected was synchronized with numerous periodic inputs indicated by the clear peaks and troughs of the curve (Figure 3F2). We examine the variability by looking at the shaded and can see that both networks had low variability in $V S$ (Figure $3 \mathrm{~F} 1 \& 3 \mathrm{~F} 2$ ) - likely owning to low levels of heterogeneity between cells. This analysis 
Figure 4 - Examples of spiking variability with stimulus. Left column: networks without oscillations. Right column: networks with oscillations $(10 \mathrm{~Hz}-5 \mathrm{~mA})$. A. Post-stimulus time histogram (PSTH) for the same network as Figure 3 with a stimulus presented. B. PSTH for the same network as A with a stimulus presented.

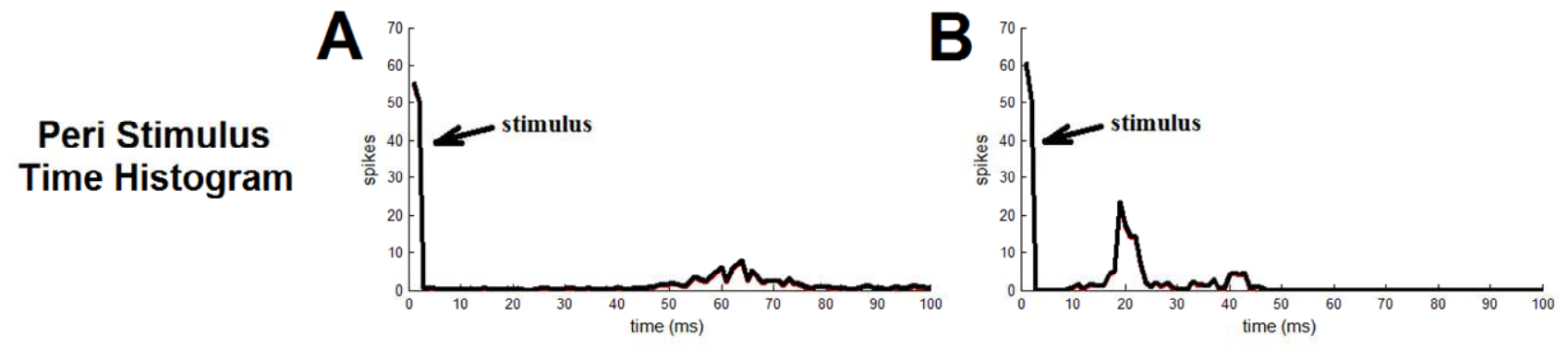

shows how quantifying the level of synchrony to a periodic signal can be useful to show the periodicity in the cells and variability between cells or neuronal networks.

In a final series of analyses we examine the poststimulus time histograms (PSTHs). For clarity, we look at spiking activity for two networks: [1] without oscillations and a stimulus (red circles - Figure 2E); and, [2] with an oscillation and a stimulus $(10 \mathrm{~Hz}, 5 \mathrm{~mA}$ - red circles - Figure 2F). For each network, the same randomly generated stimulus is presented at $1500 \mathrm{~ms}$ intervals at rising phase of the oscillating network, and post-stimulus spikes (i.e., gray box - Figure 1D) were stored to generate PSTHs. Results show that for the network without oscillations, post-stimulus spike times varied widely across time (Figure 4A). By comparison, for networks with oscillations post-stimulus spike times were more reliable and all spiking activity occurred around the peak of the wave (i.e., after $\sim 20$ ms - Figure 4B). The PSTHs generated by examining these two networks show that cells without oscillations responded with more variability across time than those with oscillations injected (compare Figure 4A versus 4B). Note that these results are consistent with the spike rasters presented in Figure 2E and 2F: [1] for the network without oscillations there was a slow and low amplitude response; [2] the network with oscillations injected responded with two high amplitude bursts of activity shortly after stimulus presentations - this is likely because the stimulus was presented during the rising phase of the oscillations when most of the neurons spike in a synchronized fashion. The variability in the PSTH can be examined by looking at the shaded red area, here we see very little variability in both networks (Figure 4A \& 4B) - possibly because the stimulus reduced the variability in spiking activity. This example is useful in depicting that PSTHs can show differences in the responses to stimuli between networks or single cells.

\section{Matlab Functions and Example Data}

The above analyses have been written as functions and can be implemented through the Matlab environment (The MathWorks, Natick, Massachusetts, United States). The functions as well as the example data file are available for download from the journal's website (www.tqmp.org). This tutorial assumes that the researcher has some experience with MATLAB: please refer to "MATLAB Primer 2013" (The MathWorks, Natick, Massachusetts, United States) for information on how to: manipulate the user interface, set the path, load data, and run functions. Go to the 'Command Line' in Matlab and run the functions using one of the example matrices (or your own data) as input. Please refer to Table 1 for file names and syntax to call the functions. All of the functions require $\mathbf{X}$, a matrix of spiking activity where rows represent neurons, columns represent time (measured in $\mathrm{ms}$ ) and within the matrix ' 1 's represent spikes; '0's depict the quiescent state. $\mathbf{X}$ is not a variable in the file; instead $\mathbf{X}$ must be generated by calling another variable (i.e., $\mathrm{X}=$ var;). Within the file 'varDEMO.mat' there are four matrices of spiking data: [1] a neural network without oscillations (see variable 'M1_withoutOSCIL'); [2] a neural network with oscillations (see 'M1_synchronous'); [3] a neural network without oscillation; but with stimulus (see 'M2_withoutOSCIL'); [4] a neural network with oscillations and stimulus (see 'M2_synchronous'). There are some functions, however, that require additional inputs (see Table 1). For instance, any analyses that compare spiking activity to periodic input need to have frequency $(\mathrm{Hz})$ as a second input. In addition, the autocorrelation script needs $\tau$ input (the size of the window around $t_{0}$ ). The plots 
Table 1 - Spiking Variability Functions

\begin{tabular}{cccc}
\hline Analysis & File Name & Input & Out \\
\hline Spike rate and Fano factor & 'spikeRTnFano' & $\mathbf{X}$ & $R, F$ \\
ISIs and Cv & 'ISInCv' & $\mathbf{X}$ & ISI, $C \boldsymbol{V}$ \\
ISIs and Lv & 'ISInLv' & $\mathbf{X}$ & ISI, $L \boldsymbol{V}$ \\
Autocorrelation & 'autocorrelation' & $\mathbf{X}, \tau$ & $\boldsymbol{r}$ \\
Period Histogram & 'periodHISTO' & X, Frequency (Hz) & $\boldsymbol{P}$ \\
Vector Strength & 'synchVECS' & X, Frequency (Hz) & $\boldsymbol{V}, \boldsymbol{P}$ \\
PSTH & 'PSTH' & X, stimUP*, stim_lag* & PSTH \\
\hline
\end{tabular}

Note: These functions are written for implementation in Matlab and can be downloaded (see

Supplement Information). Bold text denotes matrices. * marks variables that are already loaded into the file named 'varDEMO.mat'.

throughout the discussion cannot be produced by simply running the functions, they need to be created this was done so that the functions did not need to be edited (i.e., removing plotting functionality for the analysis of large datasets). The data, however, is the same and therefore the plots can be replicated.

\section{Conclusion}

Measurements of spiking variability have enhanced our understanding of how the brain may process information. In this contribution we have shown that the measurement of spiking variability can be a simplified process that enables researchers to quickly and efficiently analyse large data sets. We have presented several ways to analyze this variability, many of the measures are qualitatively unique and offer a different perspective on neuronal activity. We have shown how several measures of spiking variability can be used to characterize the behaviour of cells or networks. Several examples of variability were reviewed, for example: spike rates, synchrony, periodicity, and temporal delays. Depending on how these measures are used different variability can be quantified or visualized, for instance: across trails or repeated stimuli; or across cells in a network. Finally, we have presented functional algorithms for each analysis and example data to aid in understanding how these measures might be used.

\section{Acknowledgements}

This work benefitted from discussion with members of the Qualitative Investigation of Brain and Behaviour group (www.QUIBB.ca). We would especially like to thank Sylvain Chartier and Laurence Morissette for helpful discussions, as well as James McCorriston and Elise Bonnema for their help in developing the computational model. JPT was funded by the NSERC Discover Grant and CIHR operating funds.

\section{References}

Adler, A., Finkes, I., Katabi, S., Prut, Y., \& Bergman, H. (2013). Encoding by synchronization in the primate striatum. The Journal of Neuroscience, 33(11), 4854:4866. doi: 10.1523/JNEUROSCI.4791-12.2013

Ainsworth, M., Lee, S., Cunningham, M.O., Traub, R.D., Kopell, N.J., \& Whittington, M.A. (2012). Rates and rhythms: A synergistic view of frequency and temporal coding in neuronal networks. Neuron, 75, 572-583. doi: 10.1016/j.neuron.2012.08.004

Bosman, C.A., Schoffelen, J.M., Brunet, N., Oostenveld, R., Bastos, A.M., \& Wornelsdorf, T. (2012). Attentional stimulus selection through selection synchronization between monkey visual areas. Neuron, $\quad 75, \quad 875-888 . \quad$ doi: 10.1016/j.neuron.2012.06.037

Chang, E.F., Bao, S., Imaizumi, K., Schreiner, C.E., \& Merzenich, M.M. (2005. Development of spectral and temporal response selectivity in the auditory cortex. PNAS, 102, 16460-16465. doi: 10.1073/pnas.0508239102

Churchland, M.M., \& Abbott, L.F. (2012). Two layers of neural variability. Nature Neuroscience, 11:15.

Churchland, M.M., Yu, B.M., Cunningham, J.P., Sugrue, L.P., Cohen, M.R., \& Corrado, G.S., et al. (2010). Stimulus onset quenches neural variability: a widespread cortical phenomena. Nature Neuroscience, 13:3, 369:378. doi: 10.1038/nn.2501

Cohen, M.R., \& Newsome, W.T. (2010). What electrical microstimulation has revealed about the neural basis of cognition. Current Opinions in Neurobiology, 14, pp. 1-9.

Dayan, P., \& Abbott, L.F. (2001). Theoretical 
Neuroscience. The MIT Press: London, England.

Dupret, D., O'Neill, J., Csicsvari, J. (2013). Dynamic reconfiguration of hippocampal interneuron circuits during spatial learning. Neuron, 78, 1-15. doi: 10.1016/j.neuron.2013.01.033

Gabbiani, F., \& Cox, S.J. (2010). Mathematics for Neuroscientists. Academic Press: London, England.

Gerstner, W., \& Kistler, W. (2008). Spiking Neuron Models. Cambridge University Press: Cambridge, England.

Goldberg, J.M., \& Brown, P.B. (1969). Response of binaural neurons of dog superior olivary complex to dichotic tonal stimuli: Some physiological mechanisms of sound localization. Journal of Neurophysiology, 32, 613-636.

Hebb, D. O. (1949). The organization of behaviour. New York: Wiley \& Sons.

Hubel, D.H., \& Wiesel, T.N. (1959). Receptive fields of single neurons in the cat's striate cortex. Journal of Physiology, 148, 579-591.

Ikeda, K. (2005). Information geometry of interspike intervals in spiking neurons. Neural Computation, 17, 2719-35. doi: 10.1162/089976605774320593

Kayser, C., Montemurro, M.A., Logothetis, N.K., \& Panzeri, S. (2009). Spike-phase coding boosts and stabilizes information carried by spatial and temporal spike patterns. Neuron, 61, 597-608. doi: 10.1016/j.neuron.2009.01.008

Kuebler, E.S., Bonnema, E., McCorriston, J., Thivierge, J.P. (2014). Stimulus discrimination in networks of spiking neurons. Proceedings of the IEEE International Joint Conference on Neural Networks. Dallas, Texas.

Lei, H., Reisenman, C.E., Wilson, C.H., Gabbur, P., \& Hildebrand, J.G. (2011). Spiking patterns and their functional implications in the antennal lobe of the Tobacco Hornworm Manducta sexta. PLoS ONE, 6(8), e23382. doi: 10.1371/journal.pone.0023382

Lemon, J. (2014). The analysis of event rates using intervals. Tutorials in Quantitative Methods for Psychology, 10, 68-76.

Litwin-Kumar, A., \& Doiron, B. (2012). Slow dynamics and high variability in balanced cortical networks with clustered connections. Nature Neuroscience, 15, 1498-1505. doi: 10.1038/nn.3220

Logothetis, N.K., Augath, M., Murayama, Y., Rauch, A., Sultan, F., \& Goense, J., et al. (2010). The effect of electrical microstimulation on cortical signal propogation. Nature Neuroscience, 13, pp.12831291.
London, M., Roth, A., Beeren, L., Hausser, M., \& Latham, P.E. (2010). Sensitivity to perturbations in vivo implies high noise and suggests rate coding in cortex. Nature, 466(7302), 123-127. doi: 10.1038/nature09086

Mainen, Z.F., \& Sejnowski, T.J. (1995). Reliability of spike timing in neocortical neurons. Science, 268, 1503-1506.

Pennini F., \& Plastino, A. (2010). Diverging Fano factors. Journal of Physics: Conference Series, 246.

Ponce-Alvarez, A., Kilavik, B.E., \& Riechle, A. (2010). Comparison of local measures of spike time irregularity and relating variability to firing rate in motor cortical neurons. Journal of Computational Neuroscience, 29, 351-365. doi: 10.1007/s10827009-0158-2

Pouget, A., Dayan, P., \& Zemel, R. (2000). Information processing with population codes. Nature Neuroscience, 1, 125-132.

Reike, F., Warland, D., van Steveninck, R.R., \& Bialek, W. (1999). Spikes: Exploring the Neural Code. MIT Press: US.

Shinomoto, S., \& Shima, K. (2003). Differences in spiking patterns among cortical neurons. Neural Computation, 15, 2823-2852.

The Math Works. (2013). MATLAB Primer R2013a. retrieved from: http://www.mathworks.com/ help/pdf_doc/matlab/getstart.pdf

Thivierge, J.-P. (2008). Neural diversity creates a rich repertoire of brain activity. Communicative \& Integrative Biology, vol. 1:2.

Thivierge, J.-P., \& Cisek, P. (2008). Nonperiodic synchronization in heterogeneous networks of spiking neurons. The Journal of Neuroscience, 28(32), pp. 7968-7978. doi: 10.1523/JNEUROSCI.0870-08.2008

Toni, N., Laplagne, D.A., Zhao, C., Lombardi, G., Ribak, C.E., Gage, F.H., et al. (2008). Neurons born in the adult dentate gyrus form functional synapses with target cells. Nature Neuroscience, 11:8. doi: 10.1038/nn.2156

Turesson, H.K., Logothetis, N.K., \& Hoffman, K.L. (2012). Category-selective phase coding in the superior temporal sulcus. PNAS, 109, 19438-19443. doi: 10.1073/pnas.1217012109

Wallace, E., Benayoun, M., van Drongelen, W., \& Cowan, J.D. (2011). Emergent oscillations in networks of stochastic spiking neurons. PLOS ONE, 6, e14804. doi: 10.1371/journal.pone.0014804.

The Quantitative Methods for Psychology 
van Steveninck, R.R., Lewen, G.D., Strong, S.P., Koberle,

Variability in Neural Spike Trains. Science, 375.

R., \& Bialek, W. (1997). Reproducibility and

\section{Citation}

Kuebler, E. S., \& Thivierge, J.-P.. (2014). Spiking variability: Theory, measures and implementation in Matlab. The Quantitative Methods for Psychology, 10 (2), 131-142.

Copyright (C) 2014 Kuebler \& Thivierge. This is an open-access article distributed under the terms of the Creative Commons Attribution License (CC BY). The use, distribution or reproduction in other forums is permitted, provided the original author(s) or licensor are credited and that the original publication in this journal is cited, in accordance with accepted academic practice. No use, distribution or reproduction is permitted which does not comply with these terms.

Received: 7/09/13 Accepted: 23/02/14 